Pacific Journal of Mathematics

METRIC FAMILIES 


\section{METRIC FAMILIES}

\section{J. F. MCCLENDON}

A (continuous) metric family is a disjoint collection of metric spaces whose metrics are compatible with a given topology on the disjoint union. The purpose of this paper is to give some examples of these objects and to develop some of their basic properties. Most theorems about metric spaces can at least be formulated for metric families - some are true, some are true only with extra hypotheses, and some are false. Examples of each kind will be given. The main positive results are a version of Dugundji's extension theorems, a cross section theorem, a generalization of one of Michael's selection theorems, and a generalization of one of Coban's selection theorems.

Here a rough description of the results described above will be given. First consider the following diagram:

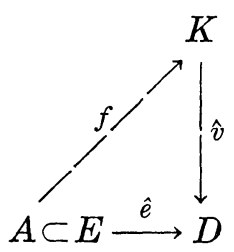

Here $\hat{e}: E \rightarrow D$ is a metric family and $\hat{v}: K \rightarrow D$ is a sub-family of a vector family. It is shown (Theorem 3.3) that under certain conditions $f$ extends to a continuous $F: E \rightarrow K$ with $\hat{v} F=\hat{e}$. If $D$ is a point then the result coincides with the extension theorem of Dugundji $[5,6]$.

Now consider the following diagram.

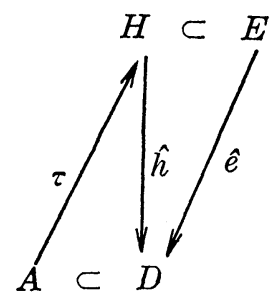

Here $\hat{h}: H \rightarrow D$ is a sub-family of a vector family $E \rightarrow D$. $\tau$ is a partial cross section of $\hat{h}$. Theorem 4.2 is a fairly general theorem giving hypotheses on $E, H$, and $\tau$ which guarantee that $\hat{h}$ has a cross section which extends $\tau$. The result is sufficiently general to 
include the Tietze extension theorem. Subsequent results specialize Theorem 4.2 and give conditions on $\hat{e}$ and $H$ which guarantee a cross-section-extension for any $\tau$.

Finally, consider the following diagram.

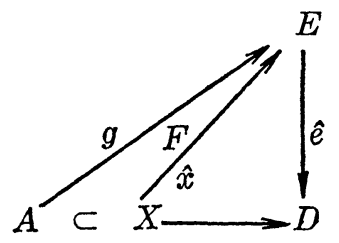

Here $\hat{e}: E \rightarrow D$ is a vector family and $F: X \rightarrow E$ is a multivalued function such that $\hat{e} F=\hat{x} . \quad g$ is a partial selection for $F$. Theorem 5.1 gives conditions on $\hat{e}$ and $F$ which permit $g$ to be extended to a continuous selection for $F$. If $D$ is a point then this becomes one of Michael's selection theorems [9]. In general the problem presented by the diagram can be thought of as a lifting problem with extra condition. The lifting problem (take $A$ empty for simplicity) is to find $f: X \rightarrow E$ with $\hat{e} f=\hat{x}$ and is the type of problem often studied in algebraic topology when $\hat{e}$ is fibration. The extra condition is to make $f$ a selection for $F$. Of course the problem can be reformulated as a pure lifting problem (not for fibrations) or a pure selection problem (not metric valued). One can also let $X_{d}=\hat{x}^{-1}(d)$ and $F_{d}=F \mid X_{d}$. Then $F$ can be thought of as a family of multifunctions with parameter space $D$. In Theorem $5.5 \hat{e}: E \rightarrow D$ is simply a metric family but the resulting selection-extension is not necessarily continuous. If $E$ is a single metric space the theorem coincides with one of Coban [3].

I wish to thank C. Himmelberg for some helpful lectures and conversations.

1. Definitions and examples. We will use the following terminology. A space is a topological space. A map is a continuous function. If $p: E \rightarrow D$ is a map then a section of $p$ is a map $s: D \rightarrow E$ with $p s=i d_{D}: D \rightarrow D$. A local section of $p$ is a map $s: V \rightarrow E, V$ open in $D$, with $p s=i d_{V}: V \rightarrow V$. Now suppose $p: E \rightarrow D$ is a given map.

$$
\begin{aligned}
E_{d} & =p^{-1}(d) \\
E_{A} & =p^{-1}(A) \\
E^{*} & =E \times_{D} E=\left\{\left(e, e^{\prime}\right) \in E \times E \mid p e=p e^{\prime}\right\} \subset E \times E \\
& =\bigcup_{d \in D} E_{d} \times E_{d} \text { (a disjoint union) } \\
S \subset E & , S_{d}=S \cap E_{d}, S_{A}=S \cap E_{A}
\end{aligned}
$$


Definition $^{1}$ 1.1. $(E, p, D, \rho)$ is a metric family if $p: E \rightarrow D$ is a map, $\rho: E^{*} \rightarrow R$ is a map, and $\rho \mid E_{d} \times E_{d}$ is a metric for each $E_{d}, d \in D$.

Usually we will simply say that $E$ is a metric family or that $E$ is a metric family over $D . \rho$ can also be called a fiber metric and $E$ can be called a fiber metric space. Later it will be convenient to use $\hat{e}: E \rightarrow D$ rather than $p: E \rightarrow D$ when more than one family is involved.

EXAMPle 1.2. Let $E$ be a metric space with metric $d$ and $p: E \rightarrow D$ any map. Then $d \mid E^{*}=\rho$ makes $E \rightarrow D$ a metric family.

ExAmple 1.2'. Let $E \rightarrow D$ be a metric family and $E \rightarrow D=$ $E \rightarrow B \rightarrow D$. Then $E \rightarrow B$ is a metric family by $E \times{ }_{B} E \subset E \times{ }_{D} E \rightarrow$ $R$. If $D$ is a point then this is 1.2 .

EXAMPLE 1.3. Let $D$ be any space and $(M, \alpha)$ any metric space. Let $E=D \times M$ and $p: E \rightarrow D$ be the natural projection. Here $E^{*}=\left\{\left(d, m, d, m^{\prime}\right) \mid d \in D, m, m^{\prime} \in M\right)$ and $E^{*}$ is homeomorphic to $D \times M \times M$. Define $\rho: E^{*} \rightarrow R$ by $\rho\left(d, m, d, m^{\prime}\right)=\alpha\left(m, m^{\prime}\right)$. That is,

$$
E^{*} \stackrel{\text { proj. }}{\longrightarrow} M \times M \stackrel{\alpha}{\longrightarrow} R \text {. }
$$

Then $E$ is a metric family. Call $E$ a product family.

EXAMPLE 1.4. Let $E \rightarrow D$ be a metric family and $S \subset E$. Then $S \rightarrow D$ is a metric family with metric obtained by restricting that of $E$.

Definition 1.5. Let $p: E \rightarrow D$ and $p^{\prime}: E^{\prime} \rightarrow D$ be metric families. A map $f: E \rightarrow E^{\prime}$ such that $p^{\prime} f=p$ is an isometry if each $f_{d}: E_{d} \rightarrow$ $E_{d}^{\prime}$ is an isometry (not necessarily onto) and $f$ is an embedding (=homeomorphism onto its image).

The question arises as to which metric families are isometrically embeddable in a product family.

EXAMPLE 1.6. Let $D=R$. Let $E$ as a set be $R \times R$ with the topology generated by usual opens and the following set

$$
S=R \times R-\{(s, t) \mid t=0,-\infty<s<0 \text { or } 0<s<+\infty\}
$$

1 This should be compared to corresponding notions of J. Dauns and K. H. Hofmann, Representation of rings by sections, Memoirs of the A. M.S. no. 83, 1968, and J. M. G. Fell, An extension of Mackey's method to Banach *-algebraic bundles, Memoirs of the A. M. S. no. $90,1969$. 
$p: E \rightarrow D$ is $p(s, t)=s . \quad \rho: E^{*} \rightarrow R$ is $\left(s, t, s, t^{\prime}\right)=\left|t-t^{\prime}\right|$. Then $(E, p, D, \rho)$ is a metric family. $E$ is not regular since the closed set $A=\{(s, t) \mid t=0,0<s<\infty\}$ and the point $(0,0)$ cannot be put into disjoint open sets. If $E$ were embeddable in a product family we would have

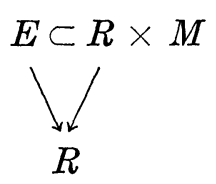

with $M$ metric-but this would imply that $E$ is regular,

ExAMPLE 1.7. Let $\mathscr{B}=\left(p: E \rightarrow B, G, F, \mathscr{A}=\left\{\left(V, h_{V}\right)\right\}\right)$ be a fiber bundle in the sense of [Steenrod, 11]. That is, $p$ is $F$-locally trivial, $G$ a topological group, $F$ is an effective left $G$-space and $\mathscr{A}$ is a $G$ atlas (really, $\mathscr{B}$ is a coordinate bundle but it determines a unique fiber bundle). Suppose now that $\alpha$ is a metric for $F$ and that the $G$ action preserves the metric, i.e., $\alpha\left(g f, g f^{\prime}\right)=\alpha\left(f, f^{\prime}\right)$. Then call $\mathscr{B}$ a metric bundle. Then $E \rightarrow B$ is a metric family since $E_{V} \leftrightarrow V \times F$ is (Example 1.3) and the equivariance assumption permits us to piece these together to form a metric family.

Recall [e.g. Husemoller, 7] a space $X$ is a $G$-space, $G$ a topological group, if there is a continuous function $G \times X \rightarrow X$, $(g, x) \rightarrow g x$, such that $1 x=x$ and $g\left(g^{\prime} x\right)=\left(g g^{\prime}\right) x$ all $g, g^{\prime} \in G, x \in X$. It is called a free $G$-space (or a fixed-point-free $G$-space) if $g x=x$ for some $x$ implies $g=1$. It is calld an effective $G$-space if $g x=x$ for all $x$ implies $g=1$. A free $G$-space is a Cartan principal $G$ space if the function $u: X^{*} \rightarrow G$ uniquely defined by $u\left(x, x^{\prime}\right) x^{\prime}=x$ is continuous. Here $p: X \rightarrow X / G=D$ is the quotient map and $X^{*}=$ $X \times_{D} X$.

THEOREM 1.8. If $G$ is a metrizable topological group and $p: X \rightarrow$ $X / G$ a Cartan principal G-space then $Y$ is a metric family.

Proof. Recall [e.g., Montgomery-Zippen, 10] that $G$ has a left invariant metric $\alpha$, so $\alpha\left(g g_{1}, g g_{2}\right)=\alpha\left(g_{1}, g_{2}\right)$ all $g_{1}, g_{2} \in G$. Define $\rho: X^{*} \rightarrow R$ by $\rho\left(x, x^{\prime}\right)=\alpha\left(u\left(g, g^{\prime}\right), 1\right)$. Then $\rho$ is continuous and we need only show that it gives a metric on each fiber. The function $u$ always satisfies the following conditions: $u\left(x, x^{\prime}\right)=1 \Leftrightarrow x=x^{\prime}$, $u\left(x, x^{\prime}\right) u\left(x^{\prime}, x^{\prime \prime}\right)=u\left(x, x^{\prime \prime}\right), u\left(x^{\prime}, x\right)=u\left(x, x^{\prime}\right)^{-1}$. The first shows that $\rho\left(x, x^{\prime}\right)=0 \Leftrightarrow x=x^{\prime}$. Also $\rho\left(x, x^{\prime}\right)=\alpha\left(u\left(x, x^{\prime}\right), 1\right)=\alpha\left(1, u\left(x, x^{\prime}\right)^{-1}\right)=$ $\alpha\left(1, u\left(x^{\prime}, x\right)\right)=\alpha\left(u\left(x^{\prime}, x\right), 1\right)=\rho\left(x^{\prime}, x\right)$. The triangle inequality is also easy to check. 
CoRollary 1.9. $G$ a topological group and $H$ a metrizable subgroup. Then $G \rightarrow G / H$ is a metric family.

Proof. If $H$ is any subgroup of $G$ then $G \rightarrow G / H$ is a Cartan principle fiber space so the result follows from 1.8 .

Let $X \rightarrow X / G$ be a Cartan principle $G$-space and $F$ a $G$-space. Then $X \times F$ is a $G$-space (diagonal action) and there is a natural map $(X \times F) / G \rightarrow X / G$ called a Cartan fiber space.

THEOREM 1.10. Suppose $F$ has a G-invariant metric. Then a Cartan fiber space is a metric family.

Proof. Similar to 1.8 (which is a special case of 1.10 ).

Note that 1.10 actually includes Example 1.7 since a Steenrod fiber bundle is homeomorphic cover the base to a (locally trivial) Cartan fiber space. In general a Cartan fiber space need not have a local cross section as the case $G \rightarrow G / H$ shows.

Recall [Atiyah, 1] that $p: E \rightarrow D$ is a vector family if each $E_{d}$ is a vector space (over $R$ here, but the general case is similar) and the functions

$$
\begin{aligned}
E \times_{D} E \longrightarrow E & \left(e, e^{\prime}\right) \longrightarrow e-e^{\prime} \\
R \times E \longrightarrow E & (t, e) \longrightarrow t e
\end{aligned}
$$

are continuous. $p: E \rightarrow D$ need not have a continuous section. However, if it does then the zero section $d \rightarrow 0_{d}$ will then be continuous.

Definition 1.11. Let $E \rightarrow D$ be a vector family. Suppose $|\cdot|: E \rightarrow R(\mathrm{e} \rightarrow|e|)$ is a function such that

(a) it is a norm on each $E_{d}\left(|e| \geqq 0,=0 \Leftrightarrow e=0,\left|e+e^{\prime}\right| \leqq\right.$ $\left.|e|+\left|e^{\prime}\right|,|\lambda e|=|\lambda||e|\right)$

(b) the function $|\cdot|: E \rightarrow R$ is continuous. Then $E \rightarrow D$ will be called a normed vector family.

Thus $\rho\left(e, e^{\prime}\right)=\left|e-e^{\prime}\right|$ makes the normed vector family $E$ into a metric family. Any normed vector bundle or vector bundle with metric [Atiyah, 1, p. 13] will be a normed vector family and hence a metric family.

\section{Elementary properties.}

Theorem 2.1. Let $p: E \rightarrow D$ be a map and $\rho: E \times_{D} E \rightarrow R$ a function. Then the following are equivalent.

(a) $\rho$ is continuous at $\left(e, e^{\prime}\right)$ 
(b) Given $\varepsilon>0$ there are opens $V, V^{\prime}$ of $E, e \in V, e^{\prime} \in V^{\prime}$ such that $\left|\rho\left(\bar{e}, \bar{e}^{\prime}\right)-\rho\left(e, e^{\prime}\right)\right|<\varepsilon$ for $\bar{e} \in V, \bar{e}^{\prime} \in V^{\prime}, p e=p \bar{e}^{\prime}$.

Corollary 2.2. Let $p: E \rightarrow D$ be a metric family, $e \in E, \varepsilon>0$. Then there is an open $W$ in $E$ with $e \in W$ such that $\bar{e}, \bar{e}^{\prime} \in W$, $p \bar{e}=p \bar{e}^{\prime}$ imply $\rho\left(\bar{e}, \bar{e}^{\prime}\right)<\varepsilon$.

Theorem 2.1 gives the following verbal description of a metric family: each $E_{d}$ is a metric space and if a pair of points in $E_{d}$ is topologically close to a pair of points in $E_{d}$, then the distance between the points of the first pair is close to the distance between the points of the second pair. It is possible to have each $E_{d}$ metric but $E \rightarrow D$ not a metric family (see example below). Corollary 2.2 shows that there is an $\varepsilon$-strip around each point of $E$, where $V$ is an $\varepsilon$-strip if $\operatorname{diam}\left(V_{d}\right)<\varepsilon$ for all $d \in D$. If there is a local section through $e \in E$ then there is something better than an $\varepsilon$-strip.

DEFinition 2.3. Let $p: E \rightarrow D$ be a metric family.

(1) $\varepsilon>0, A \subset E, B_{\varepsilon}(A)=B(A ; \varepsilon)=\left\{e \in E \mid A_{p(e)} \neq \phi\right.$ and $\left.\rho\left(e, A_{p(e)}\right)<\varepsilon\right\}$

(2) $\sigma: V \rightarrow E$ a local section of $p, B_{\varepsilon}(\sigma)=B(\sigma ; \varepsilon)=\{e \in E \mid p e \in V$, $\rho(e, \sigma p e)<\varepsilon\}=B_{\varepsilon}(\sigma(V))$.

Usually $B_{\varepsilon}(A)$ is not open in $E$, as the case $A=$ a point shows. However we do have the following facts.

THEOREM 2.4. Let $p: E \rightarrow D$ be a metric family and $\sigma: V \rightarrow E$ a local section of $p$ and $\varepsilon>0$.

(1) $B(\sigma ; \varepsilon)$ is open in $E$.

(2) Let $e_{0} \in B(\sigma ; \varepsilon)$. Suppose $\tau^{\prime}: W^{\prime} \rightarrow E$ is a local section of $E$ with $e_{0} \in \tau^{\prime}\left(W^{\prime}\right)$. Then there is a $\delta>0$ and an open neighborhood $W$ of pe such that $e_{0} \in B(\tau ; \delta) \subset B(\sigma ; \varepsilon)$ where $\tau=\tau^{\prime} \mid W$.

Proof. (1) Let $s: E_{V} \rightarrow E_{V}^{*}$ be defined by $s(e)=(e, \sigma p e)$. Then $s$ is continuous since $\sigma$ is and $B(\sigma ; \varepsilon)=(\rho s)^{-1}[0, \varepsilon)$.

(2) Let $p\left(e_{0}\right)=d_{0}$ and $\rho\left(e_{0}, \sigma\left(d_{0}\right)\right)=\varepsilon_{1}<\varepsilon_{2}<\varepsilon$. Let $W=$ $\left\{d \mid \rho\left(\tau^{\prime}(d), \sigma(d)\right)<\varepsilon_{2}\right\}$. Then $W=\left\{d \mid \tau^{\prime}(d) \in B\left(\sigma ; \varepsilon_{2}\right)\right\}=\tau^{\prime-1}\left(B\left(\sigma ; \varepsilon_{2}\right)\right)$ so $W$ is open in $D$ and $d_{0} \in W$. Set $\delta=\varepsilon-\varepsilon_{2}>0$. Then $e \in B(\tau ; \delta)$, $p e \in W$ implies $\rho(e, \tau p e)<\delta$ so $\rho(e, \sigma p e)<\rho(e, \tau p e)+\rho(\tau p e, \sigma p e)<\delta+$ $\varepsilon_{2}=\varepsilon$. Thus $e_{0} \in B(\tau ; \delta) \cap p^{-1}(W) \subset B(\sigma ; \varepsilon)$ proving the result.

Part (2) of the theorem shows that is $\mathscr{S}$ if a family of local sections of $p$ and there is at least one section through each $e \in E$ then the family $\{B(\sigma \mid W, \varepsilon) \mid \sigma \in \mathscr{S}, \varepsilon>0, W$ open, $W \subset \operatorname{dom} \sigma\}$ is a basis for a topology on $E$. Call $\mathscr{S}$ a full family of local sections of $p$ if it contains at least one local section through each point of $E$. 
DeFinition 2.5. Let $p: E \rightarrow D$ be a metric family and $\mathscr{S}$ a family of local sections of $p$. The coarse $\mathscr{S}$-topology on $E$ is the topology sub-generated by $\mathscr{S}^{\prime}=\{B(\sigma \mid W, \varepsilon) \mid \varepsilon>0, \sigma \in \mathscr{S}, W$ open, $W \subset \operatorname{dom} \sigma$ ). If $\mathscr{S}$ consists of all local cross sections of $p$ then call the resulting topology the coarse topology of $E$. A function $h: E \rightarrow Z$ is coarsely continuous (open, closed, etc.) if it is continuous (open, closed, etc.) for the coarse topology on $E$. If the topology on $E$ is the same as the coarse topology then $E$ will be called a coarse metric family.

Example 1.6 shows that a metric family may have many local sections and still not be a coarse metric family. A metric family may not have any sections (or even local sections) but when it does the set of sections has a natural metric (possibly infinite valued). More generally, consider

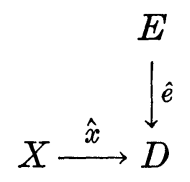

$\hat{e}: E \rightarrow D$ a metric family

Definition 2.6. $C_{D}(X, E)=\{f: X \rightarrow E \mid f$ continuous, $\hat{e} f=\hat{x}\} . \quad$ If $f, g \in C_{D}(X, E)$ then $d(f, g)=\sup \{\rho(f(x), g(x)) \mid x \in X\} \in[0,+\infty]$. If $\hat{x}=i d: D \rightarrow D$ write Sect $\hat{e}$ for $C_{D}(D, E)$.

THEOREM 2.7. Suppose $E$ has the coarse S-topology for some full family of local sections $\mathscr{S}$. If each $E_{d}$ is complete then so is $C_{D}(X, E)$.

Proof. Let $\left\{f_{n}\right\}$ be a Cauchy sequence in $C_{D}(X, E)$. Then each $\left\{f_{n}(x)\right\}$ is Cauchy so $f_{n}(x) \rightarrow f(x)$. Now let $f\left(x_{0}\right) \in B(\sigma ; \varepsilon)$ where $\sigma: V \rightarrow$ $E$ is a local section from $\mathscr{S}$ and $\hat{x}\left(x_{0}\right)=d_{0}, \sigma\left(d_{0}\right)=f\left(x_{0}\right)$ The usual argument shows that there is an $N$ such that $n>N$ implies $\rho\left(f_{n}(x), f(x)\right)<\varepsilon / 2$. Select such an $n$ so $f_{n}\left(x_{0}\right) \in B(\sigma, \varepsilon / 2)$ and there is an open set $W$ in $X$ with $x_{0} \in X$ and $f_{n}(W) \subset B(\sigma ; \varepsilon / 2)$. If $x \in W$ then $\rho(f(x), \sigma \hat{x} x) \leqq \rho\left(f(x), f_{n}(x)\right)+\rho\left(f_{n}(x), \sigma \widehat{x}(x)\right)<\varepsilon / 2+\varepsilon / 2=\varepsilon \quad$ so $f(W) \subset B(\sigma ; \varepsilon)$. Thus $f$ is continuous and by the proof $f_{n} \rightarrow f$ in $C_{D}(X, E)$.

We now consider briefly separation properties and some examples. Let us say that a map $p: E \rightarrow D$ is a Hausdorff [normal] family if for any two distinct points $x, y \in E_{D}$ [disjoint closed sets $A, B$ of $E_{d}$ ] there are disjoint open sets $V, V^{\prime}$ of $E$ with $x \in V, y \in V^{\prime}[A \subset V$, $B \subset V^{\prime}$. 
Note that if $E$ is a Hausdorff family and $D$ a Hausdorff space then $E$ is a Hausdorff space. However if $D$ and $Y$ are normal spaces then $D \times Y$ is a normal family but not necessarily a normal space. It is easy to see that a metric family is a Hausdorff family.

EXAMPLE 2.9. A metric family need not be a normal family. Note first the following general fact. Suppose $X$ a Hausdorff space and $A$ a closed subspace with metric $d: A \times A \rightarrow R$. Define $D=$ $X / A$ and $\rho: X \times_{D} X \rightarrow R$ by $\rho \mid A \times A=d$ and $\rho \mid \Delta(x) \equiv 0$. Then $(X \rightarrow X / A, \rho)$ is a metric family.

Now take $X=I \times I$ as a set with $A=I \times 0, X^{\prime}=I \times(0,1]$. Give $X^{\prime}$ the $R^{2}$ usual topology and take as a basis at $(x, 0)$ all $U_{\varepsilon}=\left[B_{\varepsilon}(x, 0) \cap X^{\prime}\right] \cup\{(x, 0)\}$. Let $B=[0,1 / 2] \times 0, B^{\prime}=(1 / 2,1] \times 0$. Then $B$ and $B^{\prime}$ are closed in $A$ but can not be separated by disjoint opens in $X$. Thus $X \rightarrow X / A$ is a metric family which is not a normal family (not even a regular family).

EXAMPLE 2.10. It is possible to have each $E_{d}$ metric but $E \rightarrow D$ not a metric family. Let $E=\left\{a_{1}, a_{2}, b_{1}, b_{2}\right\} \rightarrow D=\{a, b\}$. The opens of $E$ are $\left\{a_{1}, b_{2}\right\},\left\{a_{2}, b_{2}\right\},\left\{b_{1}\right\},\left\{b_{2}\right\},\left\{a_{1}, b_{1}, b_{2}\right\},\left\{b_{1}, b_{2}\right\},\left\{a_{2}, b_{1}, b_{2}\right\}$ and $E, \phi$. The opens of $D$ are $D, \phi$, and $\{b\} . \quad p\left(a_{i}\right)=a, p\left(b_{i}\right)=b, i=1,2$. $E \rightarrow D$ has each $E_{d}$ metric (since discrete) but $E$ is not a Hausdorff family since $a_{1}$ and $a_{2}$ can't be globally separated.

Call $p: E \rightarrow D$ is a completely regular family if for each $d \in D$ and each $A \subset E_{d}, A$ closed, $b \in E_{d}, b \notin A$, there is a function $f: E \rightarrow R$, continuous on $E$, with $f(A)=0, f(b)=1$.

EXAMPLE 2.11. Let $W$ be a Hausdorff regular space such that all coninuous functions on $W$ are constant [I wish to thank J. Porter for pointing out to me the relevance of these spaces]. Let $w_{0}, w_{0}^{\prime}$ be distinct points of $W$ and set $D=W /\left\{w_{0}, w_{0}^{\prime}\right\}$ (i.e., identify $w_{0}$ and $\left.w_{0}^{\prime}\right)$. First note that $W \rightarrow D$ is a normal family since only $A=\left\{w_{0}\right\}$ and $B=\left\{w_{0}^{\prime}\right\}$ must be separated in $W$ but $W$ is Hausdorff. Also $W \rightarrow D$ is a metric family since $\rho: W^{*} \rightarrow R, \rho\left(w_{0}, w_{0}^{\prime}\right)=1, \rho\left(w_{0}^{\prime}, w_{0}\right)=$ $1, \rho(w, w)=0$ all $w$, is a metric. Thus $W \rightarrow D$ shows that a normal family need not be a completely regular family and a metric family need not be a completely regular family.

3. Extensions. In this section we will prove a version of Dugundji's extension theorem [Dugundji, 5, 6] for metric families.

Let $V \rightarrow D$ be a vector family. A subset $K$ of $V$ is convex if each $K_{d}$ is convex $\left(k, k^{\prime} \in K_{d}\right.$ implies $t k+(1-t) k^{\prime} \in K_{d}$ for $\left.0 \leqq t \leqq 1\right)$. $V$ is locally convex if it has a basis of convex subsets. For the theorem below we can get by with somewhat less than local convexity. 
Definition 3.1. (cf. 5, p. 417,418 ). A vector family $\hat{v}: V \rightarrow D$ is of type $m$ if for every metric family $\hat{e}: E \rightarrow D$ and continuous $f: E \rightarrow V, \hat{v} f=\hat{e}$, the following is true: for each $e \in E$ and neighborhood $W$ of $f(e)$ there is a neighborhood $U$ of $e$ and a convex set $C$ of $U$ such that $f(U) \subset C \subset W$.

We study the following commutative diagram of spaces and maps.

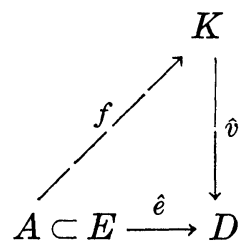

$K \subset V \rightarrow D, K$ convex, $V$ a vector family of type $m, E \rightarrow D$ a metric family.

Definition 3.2. A subset $A$ of $E$ is smooth if $\hat{e} A=D, e \rightarrow$ $\rho(e, A)$ is a continuous function $(E-A) \rightarrow R$, and the sets $B(\sigma ; \varepsilon)$ for local sections $\sigma: V \rightarrow E, \sigma(V) \subset A, \varepsilon>0$, form a basis at each point of $A$.

Theorem 3.3. Suppose $A$ is a smooth closed subset of $E$ and $E-A$ paracompact. Then $f$ extends to a map $F: E \rightarrow K$ with $\hat{v} F=\hat{e}$.

Proof. Let $e \in E-A$. Select $a_{e} \in A$ such that $\rho\left(e, a_{e}\right)<2 \rho(e, A)$. Let $\sigma=\sigma_{e}: v \rightarrow E$ be a local section such that $a_{e} \in \sigma(v) \subset A$. Define

$$
N_{e}=\left\{e^{\prime} \in E \mid \rho\left(e^{\prime}, \sigma \hat{e}\left(e^{\prime}\right)\right)<2 \rho\left(e^{\prime}, A\right)\right\}
$$

Then $N_{e}$ is an open neighborhood of $e$ since both $e^{\prime} \rightarrow \rho\left(e^{\prime}, \sigma \hat{e}\left(e^{\prime}\right)\right)$ and $e^{\prime} \rightarrow \rho\left(e^{\prime}, A\right)$ are continuous. The family of all $N_{e}$ 's is an open cover of $E-A$ so we get a locally finite subcover $\mathscr{U}=\left\{V_{\lambda} \mid \lambda \in \Lambda\right\}$ and partition of unity $\left\{\Pi_{\lambda}:(E-A) \rightarrow R\right\}$ subordinate to it. For each $\lambda$ select $e$ with $N_{e} \supset V_{\lambda}$ and define $\sigma_{\lambda}=\sigma_{e}$. Define $F: E \rightarrow K$ by

$$
F(e)= \begin{cases}f(e) & e \in A \\ \Sigma_{\lambda} \Pi_{\lambda}(e) f \sigma \hat{e}(e) & e \in(E-A)\end{cases}
$$

$F$ is continuous on $(E-A)$ by the usual argument. Let $a \in A$ and Suppose $W$ a neighborhood of $F(a)=f(a)$ in $K$. Let $U$ be a neighborhood of $a$ and $C$ a convex subset of $K$ such that $f(U \cap A) \subset C \subset W$. We may assume that $U=B(\tau ; \varepsilon)$ for a local section $\tau$ of $A \rightarrow D$ 
which passes through $a$. Let $U^{\prime}=B(\tau ; \varepsilon / 3)$. Then to prove $F$ continuous at $a$ we need only show $F\left(U^{\prime}\right) \subset W$. Let $y \in U^{\prime}$. If $y \in A$ then $F(y)=f(y) \in W$. Suppose that $y \in U^{\prime}-A$. If $y \in U_{\lambda}$ then $\rho\left(\sigma_{\lambda} \hat{e}(y), \tau \hat{e}(y) \leqq \rho\left(\sigma_{\lambda} \hat{e}(y), y\right)+\rho(y, \tau \hat{e}(y)) \leqq 2 \rho(y, A)+\rho(y, \tau \hat{e}(y))\right.$ (since $y \in U_{\lambda} \subset$ some $\left.N_{e}\right) \leqq 3 \rho(y, \tau \hat{e} y)<\varepsilon$. Thus $\sigma_{\lambda} \hat{e} y \in U$ and $f \sigma_{\lambda} \hat{e} y \in C$. Since $C$ is convex, $F(y) \in C \subset W$ showing $F\left(U^{\prime}\right) \subset W$.

Dugundji's extension theorem is the case $D=$ point since in this case every $A$ is smooth and $E$ metric so $E-A$ is paracompact. Now let $L$ be a topological vector space of type $m$ (as in [5] or the case $D=$ point in Definition 3.1 ) and $T$ a convex subset. Consider

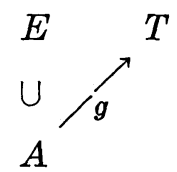

$E \rightarrow D$ a metric family.

Corollary 3.4. If $A$ is a closed smooth subset of $E$ and $E-A$ is paracompact then $g$ extends to a continuous function $G: E \rightarrow T$.

Proof. Use $V=D \times L \rightarrow D$ and $K=D \times T \rightarrow D$. Let $f=$ $(\hat{e}, g): A \rightarrow K$. The theorem gives an extension $F=(\hat{e}, G): E \rightarrow K$ and $G$ is the desired extension of $g$.

Now consider

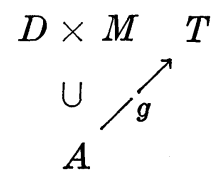

$T=$ convex subset of $L=$ vector space of type $m . \quad M=$ metric space.

Corollary 3.5. Let $A$ be a closed smooth subset of $D \times M$ and suppose $(D \times M)-A$ is paracompact. Then $g$ extends to a continuous $G: D \times M \rightarrow T$.

Just as in the case $D=$ point we can say something about linearity. Suppose $S \rightarrow D$ is any map and $V \rightarrow D$ a vector family. Let $C_{D}(\mathrm{~S}, V)$ be the set of map's $h: S \rightarrow V$ with $\hat{v} h=\hat{s}$. Then if $C_{D}(S, V)$ is not empty it has a natural vector space structure (add values). The proof of Theorem 3 gives a linear function

$$
\alpha: C_{D}(A, V) \longrightarrow C_{D}(E, V) \quad \alpha(f)=F
$$


and if $\beta: C_{D}(E, V) \rightarrow C_{D}(A, V)$ is the function $\beta G=G \mid A$ then we have $\beta \alpha=$ identity.

4. Cross sections. In this section we will study the following commutative diagram $E \rightarrow D$ a normed vector family.

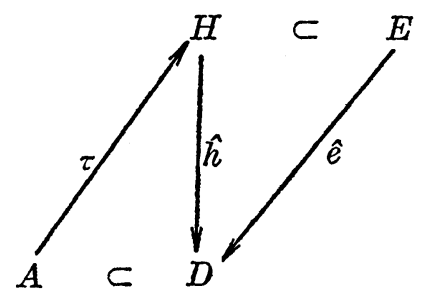

The objective is to find conditions under which $\hat{h}: H \rightarrow D$ has a cross section which extends $\tau$. If $A=\phi$ this is a pure cross section problem and by introducing an auxiliary space we can reduce the problem to a cross section problem. Define $G=G(\tau) \subset E$ by $G \cap E_{d}=$ $H_{d}$ if $d \notin A$ and $G \cap E_{d}=\{\tau(d)\}$ if $d \in A$. Thus

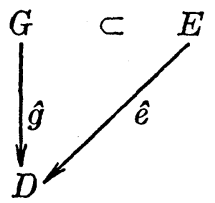

and $\hat{h}$ has a cross section extending $\tau$ iff $\hat{g}$ has a cross section.

Recall [Dold, 4] that a cover $\mathscr{C}$ of a space is numerable if there is a family $\left\{\Pi_{\lambda}: D \rightarrow[0,1]\right\}$ of continuous functions such that $\left\{\Pi_{\lambda}^{-1}(0,1]\right\}$ is locally finite and a refinement of $\mathscr{U}$ and $\Sigma_{\lambda} \Pi_{\lambda}(d)=1$ all $d \in D$.

Definition 4.1. $(H, \tau)$ is numerably sectioned in $E$ if for every $\varepsilon>0$ and every open convex $E^{\prime}$ of $E$ with $\hat{e}\left(E^{\prime} \cap G\right)=D$ there is a family $\mathscr{S}=\mathscr{S}\left(\varepsilon, E^{\prime}\right)$ of local sections of $\hat{e}$ such that

(a) $E^{\prime} \cap G \subset \bigcup\left\{B_{\varepsilon}(\sigma) \mid \sigma \in \mathscr{S}\right\}$

(b) $\mathscr{\mathscr { C }}=\mathscr{\mathscr { C }}\left(\varepsilon, E^{\prime}\right)=\left\{\hat{e}\left(B_{\varepsilon}(\sigma) \cap G \cap E^{\prime}\right) \mid \sigma \in \mathscr{S}\right\}$ is a numerable cover of $D$.

The dependence on $\tau$ of the above condition appears to be unavoidable in the general case. However, if $D$ is paracompact it can often be eliminated (see 4.9 below).

THEOREM $^{2}$ 4.2. Suppose that $\hat{e}: E \rightarrow D$ is a normed vector family

${ }^{2}$ This theorem and its corollaries are valid, with the same proof, under the weaker hypotheses: $|\cdot|: E \rightarrow R$ is upper semi-continuous and a pseudo-norm on each $E_{d}$. 
and Sect $(\hat{e})$ is complete. Suppose $H_{d}$ is closed in $E_{d}$ and convex for $d \in D-A$ and that $(H, \tau)$ is numerably sectioned in $E$. Then $\hat{h}$ has a cross section extending $\tau$.

Lemma 4.3. Suppose $\hat{e}: E \rightarrow D$ is a normed vector family, each $H_{d}$ is convex, and $(H, \tau)$ is numerably sectioned in $E$. Suppose $\varepsilon>0$ and $E^{\prime}$ open convex with $\hat{e}\left(E^{\prime} \cap G\right)=D$. Then $B\left(G \cap E^{\prime} ; \varepsilon\right) \rightarrow D$ has a cross section.

Proof. Let $\mathscr{U}=\mathscr{C}\left(\varepsilon, E^{\prime}\right), \quad\left\{\Pi_{\lambda}: D \rightarrow[0,1]\right\}$ be as in Definition 4.1. For each $\lambda \in A, \Pi_{\lambda}^{-1}(0,1] \subset V \in \mathscr{Q}$ for some $V$. Pick such a $V$ and its section $\sigma$ and let $\sigma_{\lambda}=\sigma \mid \Pi_{\lambda}^{-1}(0,1]$. Define $\sigma=\Sigma \Pi_{\lambda} \sigma_{\lambda}: D \rightarrow$ $E$. Then $\sigma$ is continuous by the usual argument. Each $\sigma_{2}$ is a local section of $B\left(G \cap E^{\prime} ; \varepsilon\right) \rightarrow D$ and $B\left(G_{d}, \varepsilon\right)$ is convex so $\sigma$ is a section of $B\left(G \cap E^{\prime} ; \varepsilon\right) \rightarrow D$.

Proof of 4.2. We will construct a sequence $\sigma_{1}, \sigma_{2}, \ldots$ of sections of $e: E \rightarrow D$ such that

$$
\begin{aligned}
& \left(A_{n}\right)(n>1) \rho\left(\sigma_{n}, \sigma_{n-1}\right)<1 / 2^{n-2} \\
& \left(B_{n}\right) \rho\left(\sigma_{n}, G\right)<1 / 2^{n} .
\end{aligned}
$$

By $(A),\left\{\sigma_{n}\right\}$ is a Cauchy sequence in Sect $\hat{e}$ and by completeness $\sigma_{n} \rightarrow \sigma$ and $\sigma$ is a continuous section of $\hat{e}$. By $(B)$ and the fact that each $G_{d}$ is closed in $E_{d}$ we see that $\sigma(D) \subset G$ so that $\sigma$ is the desired cross section.

Lemma 4.4 gives $\sigma_{1}$. Suppose that $\sigma_{1}, \sigma_{2}, \cdots, \sigma_{n}$ are defined with properties (A) and (B). The lemma gives a cross section

$$
\sigma_{n+1}: D \longrightarrow B\left(G \cap B\left(\sigma_{n}, 1 / 2^{n}\right), 1 / 2^{n+1}\right)
$$

since $\left(B_{n}\right)$ shows $\hat{e}\left(G \cap B\left(\sigma_{n}, 1 / 2^{n}\right)\right)=D$. Thus $\left(B_{n+1}\right) \rho\left(\sigma_{n+1}, \sigma_{n}\right)<$ $1 / 2^{n+1}$ and $\left(A_{n+1}\right) \rho\left(\sigma_{n+1}, \sigma_{n}\right)<1 / 2^{n-1}$ are clear (since $1 / 2^{n+1}+1 / 2^{n}<$ $\left.1 / 2^{n-1}\right)$.

It's interesting to note that the above theorem includes the Tietze extension theorem. To be precise it includes a deduction of the Tietze extension theorem from the Uryrohn theorem on the existence of certain real valued functions on a normal space.

Corollary 4.4. (Tietze 12) $X$ normal $\supset A$ closed. $f: A \rightarrow[0,1]$ a map. Then $f$ extends to a map $F: X \rightarrow[0,1]$.

Proof. Consider $\left[((X-A) \times I) \cup G_{f}\right]=G \subset X \times I \subset X \times R$

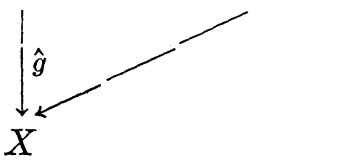


where $G_{f}$ is the graph of $f$. For $t \in I$ define $\sigma_{t}(x)=(x, t)$. These and the compactness of $I$ allow $\mathscr{S}_{\varepsilon}$ to be finite in Definition 4.1. Also $\hat{g}$ is an open map so $\mathscr{C}$ of 4.1 is a finite open cover. Urysohn's theorem permits the construction of a subordinate partition of unity [Bourbaki, 2] and the result now follows from 4.2.

Definition 4.5. $\hat{e}: E \rightarrow D$ is a Banach family if it is a normed vector family (see 1.11), has the coarse topology for a full family of local sections (see 2.5), and each $E_{d}$ is complete.

Corollary 4.6. Suppose ê: $E \rightarrow D$ is a Banach family. Suppose that each $H_{d}$ is closed in $E_{d}$ and convex and that $(H, \tau)$ is numerably sectioned in $E$. Then $h$ has a cross section extending $\tau$.

Proof. By 2.7 Sect $\hat{e}$ is complete.

Definition 4.7. $H$ is sectioned in $E$ there exists $H^{\prime}$ dense in $H$ such that for every $h \in H^{\prime}$ there is a local section $\sigma: V \rightarrow E$ of $\hat{e}$ with $h \in \sigma(V)$.

CoROLlaRY 4.8. Suppose $D$ paracompact, $\hat{e}: E \rightarrow D$ a normed vector family, Sect $\hat{e}$ complete, $\hat{h}$ coarsely open and onto. Suppose $H$ is sectioned in $E$ and each $H_{d}$ is closed and convex. Then $\hat{h}$ has $a$ cross section extending $\tau$ for any closed $A$ and partial section $\tau$.

Proof. For any $\varepsilon>0, E^{\prime}$ (as in 4.1) take $\mathscr{S}=$ all local sections of $E$. Then (a) is clear. If we can show that $\hat{g}$ is coarsely open then $\mathscr{C}$ of Definition 4.1 will be an open cover and will be numerable by the paracompactness of $D$. Let $d \in \hat{e}(B(\sigma ; \varepsilon) \cap G)$. If $d \in D-A$ then $d \in \hat{h}(B(\sigma ; \varepsilon)) \cap(D-A) \subset \hat{e}(B(\sigma ; \varepsilon) \cap G)$. If $d \in A$ select $W$ open in $D$ such that $\tau(W \cap A) \subset B(\sigma ; \varepsilon)$ and check that $d \in W \cap \hat{h}(B(\sigma ; \varepsilon) \cap H) \subset \hat{e}(B(\sigma ; \varepsilon) \cap G)$. So $\hat{g}$ is coarsely open because $\hat{h}$ is. Thus 4.2 applies.

Corollary 4.9. Suppose $D$ paracompact $\hat{e}: E \rightarrow D$ a Banach family, $\hat{h}$ open and onto, each $H_{d}$ closed and convex. Then $\hat{h}$ has $a$ cross section extending $\tau$.

Proof. This follows from 4.8. $H$ is sectioned since $\hat{e}$ has a full family of local cross sections. Sect $\hat{e}$ is complete by 2.7.

CoRollary 4.10. $D$ paracompact, $\hat{e}: E \rightarrow D$ a Banach family, $\hat{e}$ open and onto. If $A$ is any closed subset of $D$ and $\tau$ is a cross section of $\hat{e}$ over $A$ then $\tau$ extends to a cross section of $\hat{e}$. 
Note that in 4.8-4.10 the hypotheses are independent of $A$. Recall [4] that a map $\hat{t}: T \rightarrow D$ has the section extension property if each cross section over a set $A$ which extends to a halo extends to a cross section over $D . \quad V \supset A$ is a halo if there is a continuous function $\alpha: D \rightarrow[0,1]$ with $A \subset \alpha^{-1}(1)$ and $(D-V) \subset \alpha^{-1}(0)$.

Corollary 4.11. Suppose $T \rightarrow D$ satisfies the hypotheses of 4.8 or 4.9 or 4.10 , over each set of a numerable covering of $D$. Then $T \rightarrow D$ has the section extension property.

Proof. Since Dold showed that the section extension property is local $[4$, p. 229].

5. Selections. In this section some of the results of $\S 4$ are used to prove selection theorems. Call $F: X \rightarrow E$ a multifunction if it is a relation which assigns to each $x \in X$ a nonempty subset of $E$. The graph of $F=G(F)=\{(x, e) \mid e \in F(x)\} \subset X \times E$. A selection for $F$ is a single valued function $f: X \rightarrow E$ such that $f(x) \in F(x)$ for all $x \in X$. Consider

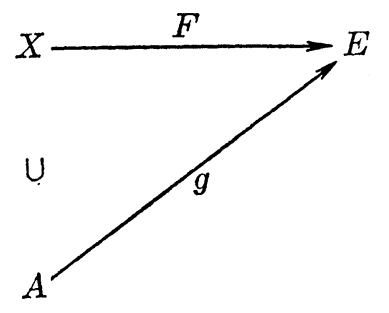

$F$ a multifunction

$g$ a continuous selection for $F \mid A$

The selection-extension problem [Michael, 9] is: can we find a continuous selection for $F$ which extends $g$ ? Here we shall study the following version

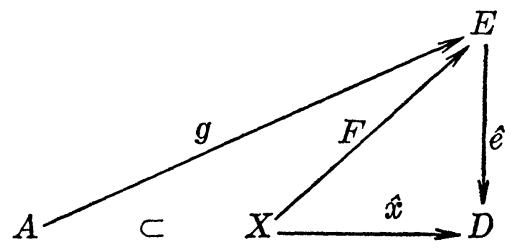

$F$ is a multifunction, $g, \hat{x}, \hat{e}$ are maps, $\hat{e} F=\hat{x}, \hat{e} g=\hat{x} \mid A$ and $g$ is a selection for $F \mid A$. The object is to find a continuous selection for $F$ extending $g$. Note that $\hat{e} f=\hat{x}$ will follow. Recall that a 
multifunction $F$ is lower semi-continuous if $F^{-1}$ of an open set is open (recall $\left.F^{-1}(S)=\left\{x \in X \mid F^{\prime}(x) \cap S \neq \varnothing\right\}\right)$.

Theorem 5.1. Suppose $X$ paracompact, $A$ closed, $\hat{e}: E \rightarrow D a$ Banach family. Suppose $F$ is lower semi-continuous and has closed, convex values. Then $F$ has a continuous selection extending $g$.

Proof. Consider the following diagram.

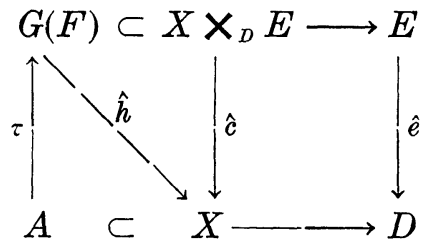

Here $X \times_{D} E=\{(x, e) \in X \times E \mid \hat{x}(x)=\hat{e}(e)\}=C . \quad C \rightarrow X$ gets the structure of a Banach family from $\hat{e}$. Specifically $(x, e)+\left(x, e^{\prime}\right)=$ $\left(x, e+e^{\prime}\right)$ is the addition, $\lambda(x, e)=(x, \lambda e)$ the scalar multiplication, $C_{x}$ is isometric with $E_{\hat{x} x}$ so is complete. If $(x, e)$ is given with $\hat{x} x=$ $d=\hat{e} e$ then there is an open $V$ of $D$ and local section $\sigma$ over $V$ through $e . \quad \sigma^{\prime}=(i d, \sigma \hat{x})$ is a local section of $\hat{c}$ over $\hat{x}^{-1}(V)$ through $(x, e)$. It is not hard to check that $\hat{h}$ is an open map since $F$ is lower semi-continuous (this is Prop. 1.2 of [3]). The hypotheses show that each $G(F)_{d}$ is closed and convex. Define $\tau(a)=(\alpha, g(a))$. Now Corollary 4.9 gives a cross section $\sigma: X \rightarrow G(F)$ of $\hat{h}$ which extends $\tau$ and the composition $X \rightarrow G(F) \subset X \times_{D} E \rightarrow E$ is the desired selection-extension.

CoRollary 5.2. [Michael, 9] $E$ a Banach space, $F: X \rightarrow E$ a lower semi-continuous multifunction with closed convex values. $X$ paracompact $\supset A$ closed and $g: A \rightarrow E$ a continuous selection for $F \mid A$. Then $F$ has a continuous selection which extends $g$.

Proof. This is the case $D=$ point of Theorem 5.1.

In [9] Michael proves several variants of 5.2. Each of these can be generalized to the metric family setting. Theorem 5.1 is a consequence of Theorem 4.9. Similarly each of the theorems of $\S 4$ gives rise to a selection theorem. Now consider the following situation.

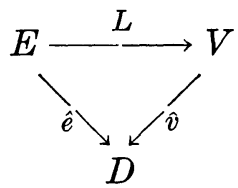

$E \rightarrow D$ a Banach family

$V \rightarrow D$ a vector family 
Corollary 5.3. Suppose $L$ is linear (i.e. each $L_{d}$ is continuous linear $-L$ is a family of linear operators), open, and onto, and $V$ $i$ s paracompact. Then $L$ has a continuous right inverse ( $M: V \rightarrow E$ such that $L M=i d)$. In fact, any partial right inverse on a closed subset $A$ of $V$ can be extended to a right inverse.

Proof. Consider

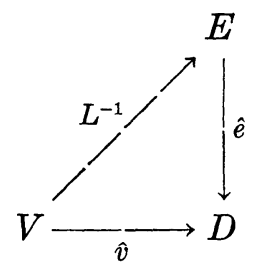

Since $L$ is open and onto, $F=L^{-1}$ is a lower semi-continuous multifunction. It has closed convex values since $L$ is linear and $V$ is Hausdorff. Theorem 5.1 gives a continuous selection for $L^{-1}$ which is a right inverse for $L$.

Until now we have looked for continuous cross-sections and selections but for our final result we will relax this restriction. Henceforth sections and selections are simply single valued functions and all continuity conditions will be stated explicitly. In [3] Coban proves a selection theorem for a multifunction from a $\sigma$-space to a complete metric space. We will generalize this to the metric family setting. Several of Coban's other results can be generalized in the same manner. A space $X$ is a $\sigma$-space if for every family $\mathscr{C}$ of open sets there is a $\sigma$-discrete refinement $\mathscr{W}$ of closed sets such that $\bigcup \mathscr{U}=\bigcup \mathscr{W}$. Coban points out $[3$, p. 275] that each of the following is a sufficient condition for a space $X$ to be a $\sigma$-space: (a) $X$ is weakly paracompact and completely normal, (b) $X$ has a $\sigma$-discrete filter, (c) $X$ is a symmetric space satisfying the first axiom of countability, (d) $X$ has a uniform structure, (e) $X$ has a refining sequence of coverings. Recall that a subset of a topological space is an $F_{\sigma}$ set if it is a countable union of closed sets. In a $\sigma$-space every difference of closed sets is an $F_{\sigma}$ set. In particular open sets are $F_{\sigma}$ sets.

Definition. 5.4. $E \rightarrow D$ a metric family, $V$ open in $E, V_{n}=$ $\{e \in E \mid \rho(e, E-V)>1 / n\} . \quad V$ is approachable if $V_{n}$ is open for all sufficiently large $n$.

Consider the following situation 


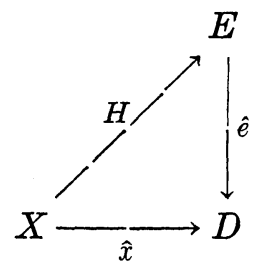

Assume that $\hat{e}$ is a metric family with the coarse topology defined by a full family of continuous local sections and $E_{d}$ complete. Suppose that $X$ is a $\sigma$-space and $H$ is a lower semi-continuous multifunction with $\hat{e} H=\hat{x}$ and closed values.

THEOREM 5.5. Under the hypotheses above $H$ has a selection $h$ such that $h^{-1}(V)$ is an $F_{\sigma}$ set for every approachable open $V$ of $E$.

Proof. For $n=1,2, \cdots$, we will find a function $h_{n}: X \rightarrow E$ with $\hat{e} h_{n}=\hat{x}$ and subsets $X_{n, 1}, X_{n, 2}, \cdots$ such that

$\left(A_{n}\right)(n>1) \rho\left(h_{n}, h_{n-1}\right)<1 / 2^{n+1}$

$\left(B_{n}\right) \rho\left(h_{n}, H\right)<1 / 2^{n-1}$

$\left(C_{n}\right) h_{n} \mid X_{n, k}$ is continuous

$\left(D_{n}\right)\left\{X_{n, k} \mid k=1,2, \cdots\right\}$ is a disjoint cover of $X$ by $F_{\sigma}$ sets.

If this has been done then define $h: X \rightarrow E$ by $h=\lim h_{n}$. $h$ is well defined by $(A)$ and the completeness of each $E_{d}$ and it is a selection for $H$ by $(B)$. Consider

$$
h^{-1}(V)=\bigcup_{n=1}^{\infty} h_{n}^{-1}\left(V_{n}\right) \quad V_{n} \text { as in Definition 5.4. }
$$

By [8, p. 398] this equation is true in each $X_{d}$ so it is true in $X$. There is no loss in generality in assuming each $V_{n}$ open so by $(C)$ and $(D)$ each $h_{n}^{-1}\left(V_{n}\right)$ is an $F_{\sigma}$ and hence $h^{-1}(V)$ is an $F_{\sigma}$ set.

Proof of $A_{n+1}-D_{n+1}$ assuming $A_{n}-D_{n}$ \{with modifications for $n=0$. Let $h_{n}, X_{n, 1}, X_{n, 2}, \cdots$, satisfy $A_{n}-D_{n}$ \{ignore this if $n=0$. Fix $k$, for now, and define $H_{k}: X_{n, k} \rightarrow E$ by $H_{k}(x)=H(x) \cap B\left(h_{n}(x)\right.$, $1 / 2^{n+1}$ ) \{if $n=0$, use $X_{0,1}=X, X_{0, k}=\phi$ for $k>1$, and $H_{1}=H$ \}. By $\left(B_{n}\right) H_{k}(x)$ is nonempty and $H_{k}$ is lower semi-continuous on $X_{n, k}$ because $h_{n}$ is continuous there. Define $\mathscr{U}=\left\{H_{k}^{-1}\left(B\left(\sigma, 1 / 2^{n+2}\right) \mid \sigma\right.\right.$ a local section of $\hat{e}\}$. Then $\mathscr{C}$ is an open cover of $X_{n, k}$ so there are discrete families of closed sets $\mathscr{W}_{p}=\left\{A_{p, \alpha} \mid \alpha \in I_{p}\right\} p=1,2, \ldots$ such that $\mathscr{W}=\bigcup \mathscr{W}_{p}$ refines $\mathscr{C}$ and covers $X_{n, k}$. If $\alpha \in I_{p}$ select $\sigma_{\alpha}$ a local section with $A_{p, \alpha} \subset H_{k}^{-1} B\left(\sigma_{\alpha}, 1 / 2^{n+2}\right)$. Thus $\rho\left(H(x), \sigma_{\alpha}(x)\right)<1 / 2^{n+2}$ for $x \in A_{p, \alpha}$. Now define 


$$
\begin{aligned}
& W_{i, k}^{\prime}=\mathbf{U} \mathscr{W}_{i} \\
& W_{i, k}=W_{i, k}^{\prime} \backslash \bigcup_{j=1}^{i-1} W_{j, k}^{\prime}=W_{i, k}^{\prime} \backslash \bigcup_{j=1}^{i-1} W_{j, k}
\end{aligned}
$$

Then $W_{i, k}$ is a difference of closed sets so an $F_{\sigma}$ subset of $X_{n, k}$, so an $F_{\sigma}$ subset of $X$. The $W_{i, k}$ 's form a disjoint cover of $X_{n, k}$ by $F_{\sigma}$ subsets of $X$. Define

$$
\begin{array}{ll}
g_{i k}^{\prime}: W_{i k}^{\prime} \longrightarrow E & g_{i k}^{\prime}=\sigma_{\alpha} \text { on } A_{i \alpha} \\
g_{i k}: W_{i k} \longrightarrow E & g_{i k}=g_{i k}^{\prime} \mid W_{i k} .
\end{array}
$$

Thus $g_{i k}^{\prime}$ is continuous on each $A_{i, \alpha}$ and $\mathscr{W}_{i}$ discrete so $g_{i k}^{\prime}$ continuous on $W_{i k}^{\prime}$ and $g_{i k}$ continuous on $W_{i k}$.

Now consider the family of all $W_{i k}, i, k=1,2, \ldots$ This is a countable family so it can be reordered and renamed as $X_{(n+1), 1)}$ $X_{(n+1), 2)} \cdots$. Hence the $X_{(n+1), k}$ form a disjoint cover of $X$ by $F_{\sigma}$ subsets of $X$ which proves $\left(D_{n+1}\right)$. Define $h_{n+1}: X \rightarrow E$ by $h_{n+1} \mid W_{i k}=$ $g_{i k}$. Then $\left(C_{n+1}\right)$ and $\left(B_{n+1}\right)$ are easily checked. For $n>1$, condition $\left(A_{n+1}\right)$ is a consequence of the definition of $H_{k}$ and $\mathscr{C}$ since $1 / 2^{n+1}+$ $1 / 2^{n+2}<1 / 2^{n}$. This completes the proof.

DEFINITION 5.6. $E \rightarrow D$ is a locally embedable metric family if for every $d \in D$ there is an open $W=W(d)$ neighborhood of $d$ and a metric space $M=M(d)$ and an isometric embedding of metric families

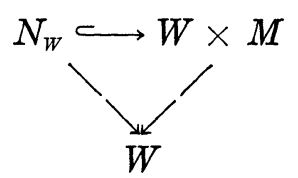

CoRollary 5.7. Suppose in 5.5 that $E$ is a locally embedable metric family. Then $H$ has a selection $h$ such that $h^{-1}(V)$ is an $F_{o}$ set for all $V$ in some basis of $E$.

Proof. In $W \times M$ if $V=W^{\prime} \times B \quad B=B_{\varepsilon}(m)$ then $V_{n}=W^{\prime} \times B_{n}$ is open so $V$ is approachable and sets $V \cap E_{W}$ are a basis of $E$.

DeFinition 5.8. $E \rightarrow D$ is a sub-euclidean metric family if for every $d \in D$ there is a neighborhood $W=W(d)$ of $d$ and an integer $n=n(d)$ and a closed isometric embedding of metric families.

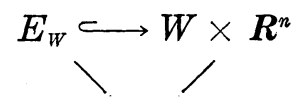


Corollary 5.9. Suppose in 5.5 that $E \rightarrow D$ is a sub-euclidean metric family. Then $H$ has a selection $h$ such that $h^{-1}(V)$ is an $F_{o}$ for every open $V$ of $E$.

Proof. Let $A$ be closed in $E$ and define $g_{A}: E \rightarrow[0,+\infty]$ by $g(e)=\rho(e, A)$. Then $V_{n}=g_{E-V}^{-1}(1 / n,+\infty]$ so we need only show that each $g_{A}$ is lower semi-continuous. We may assume $E=D \times \boldsymbol{R}^{n}$. Let $\varepsilon>0$ and $g(e)>\varepsilon$. Suppose $e=\left(d_{0}, u_{0}\right)$ and $g(e)=\alpha>\beta_{2}>\beta_{1}>\varepsilon$. Set $\gamma=\beta_{2}-\beta_{1}$. We will prove that there is a neighborhood $W$ of $d_{0}$ such that $e=(d, u) \in W \times B\left(u_{0}, \gamma\right)$ implies $g(d, u)>\varepsilon$. Suppose this is false. Then for each $W \ni d_{0}$ get $\mathrm{d} \in W, u \in B\left(u_{0}, \gamma\right)$ and $g(d, u) \leqq \varepsilon$. So we can find $u^{\prime} \in R^{n}$ with $\left(d, u^{\prime}\right) \in A, d\left(u, u^{\prime}\right) \leqq \beta_{1}$. Then $\left\{\left(d_{W}, u_{W}^{\prime}\right)\right\}$ is a net on $A,\left\{u_{W}^{\prime}\right\}$ is a net on $\overline{B\left(u_{0}, \beta_{1}+\gamma\right)}$ so has a convergent subnet converging to $\bar{u}$. But this gives a net on $A$ converging to $\left(d_{0}, \bar{u}\right)$ and $A$ is closed so $\left(d_{0}, \bar{u}\right) \in A$. Hence $g(e) \leqq$ $\rho\left(e,\left(d_{0}, \bar{\mu}\right)\right)=d\left(u_{0}, \bar{\mu}\right) \leqq \beta_{1}+\gamma=\beta_{2}<\alpha$, a contradiction.

CoRollary. Let $p: E \rightarrow X$ be a metric fiber bundle with fiber $F$ a closed subset of $\boldsymbol{R}^{n}$ and $X$ a $\sigma$-space. Then $p$ has a cross section $\sigma$ such that $\sigma^{-1}(V)$ is an $F_{\sigma}$ set for all opens $V$ of $E$.

\section{Proof. Apply 5.9 with $X=D$ and $H=p^{-1}$.}

Note that we cannot expect a continuous cross section in the setting of Corollary 5.10 as is shown by the Hopf bundle $S^{3} \rightarrow S^{2}$ with fiber $S^{1}$.

\section{REFERENCES}

1. M. F. Atiyah, K-Theory, Harvard lecture notes, 1964.

2. N. Bourbaki, General Topology II, Addison-Wesley, 1966.

3. M. M. Coban, Many-valued mappings and Borel sets I. Trans. Moscow Math. Soc., 22 (1970), 258-280 (Amer. Math. Soc. translations).

4. A. Dold, Partitions of unity in the theory of fibrations, Ann. of Math., 78 (1963), 223-255.

5. J. Dugundji, Topology, Allyn and Bacon, Boston (1966).

6. - - An extension of Tietze's theorem, Pacific J. Math., 1 (1951) 353-367.

7. D. Husemoller, Fiber Bundles, McGraw-Hill, New York (1966).

8. K. Kuratowski and C. Ryll-Nardzewski, A general theorem on selectors, Bull. Acad. Polon. Sci. Ser. Sec. Math. Astron. Phys., 13 (1965), 397-403.

9. E. A. Michael, Continuous selections I, Ann. of Math., (2) 63 (1956), 361-382.

10. D. Montgomery and L. Zippin, Topological Transformation Groups, Interscience, New York, 1955.

11. N. Steenrod, The topology of fiber bundles, Princeton, 1951.

12. H. Tietze, Uber Funktionen, die auf einer abgeschlossen Menge stetig sind, J. für die R. und Ang. Math., 145, (1915), 9-14.

Received February 8, 1974 and in revised form February 3, 1975. 



\section{PACIFIC JOURNAL OF MATHEMATICS}

\section{EDITORS}

RICHARD ARENS (Managing Editor)

University of California

Los Angeles, California 90024

\section{J. DugundJI}

Department of Mathematics University of Southern California Los Angeles, California 90007

D. Gilbarg and J. Milgram

Stanford University

Stanford, California 94305
University of Washington Seattle, Washington 98105

\section{ASSOCIATE EDITORS}
E. F. BECKENBACH
B. H. NeumanN
F. WolF
K. YoShIDA

\section{SUPPORTING INSTITUTIONS}

\author{
UNIVERSITY OF SOUTHERN CALIFORNIA \\ STANFORD UNIVERSITY \\ UNIVERSITY OF TOKYO \\ UNIVERSITY OF UTAH \\ WASHINGTON STATE UNIVERSITY \\ UNIVERSITY OF WASHINGTON \\ $\stackrel{*}{*} \stackrel{*}{*} \stackrel{*}{ }{ }^{*}$ AMERICAN MATHEMATICAL SOCIETY
}

The Supporting Institutions listed above contribute to the cost of publication of this Journal, but they are not owners or publishers and have no responsibility for its content or policies.

Mathematical papers intended for publication in the Pacific Journal of Mathematics should be in typed form or offset-reproduced, (not dittoed), double spaced with large margins. Underline Greek letters in red, German in green, and script in blue. The first paragraph or two must be capable of being used separately as a synopsis of the entire paper. Items of the bibliography should not be cited there unless absolutely necessary, in which case they must be identified by author and Journal, rather than by item number. Manuscripts, in triplicate, may be sent to any one of the editors. Please classify according to the scheme of Math. Reviews, Index to Vol. 39. All other communications should be addressed to the managing editor, or Elaine Barth, University of California, Los Angeles, California, 90024.

The Pacific Journal of Mathematics expects the author's institution to pay page charges, and reserves the right to delay publication for nonpayment of charges in case of financial emergency.

100 reprints are provided free for each article, only if page charges have been substantially paid. Additional copies may be obtained at cost in multiples of 50 .

The Pacific Journal of Mathematics is issued monthly as of January 1966. Regular subscription rate: $\$ 72.00$ a year (6 Vols., 12 issues). Special rate: $\$ 36.00$ a year to individual members of supporting institutions.

Subscriptions, orders for back numbers, and changes of address should be sent to Pacific Journal of Mathematics, 103 Highland Boulevard, Berkeley, California, 94708.

\section{PUBLISHED BY PACIFIC JOURNAL OF MATHEMATICS, A NON-PROFIT CORPORATION}

Printed at Kokusai Bunken Insatsusha (International Academic Printing Co., Ltd.), 270, 3-chome Totsuka-cho, Shinjuku-ku, Tokyo 160, Japan.

\section{Copyright (C) 1975 by Pacific Journal of Mathematics} Manufactured and first issued in Japan 


\section{Pacific Journal of Mathematics}

\section{Vol. 57, No. $2 \quad$ February, 1975}

Norman Larrabee Alling, On Cauchy's theorem for real algebraic curves with boundary .......

Daniel D. Anderson, A remark on the lattice of ideals of a Prüfer domain ..................

Dennis Neal Barr and Peter D. Miletta, A necessary and sufficient condition for uniqueness of

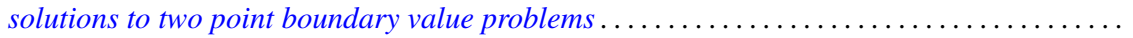

Ladislav Beran, On solvability of generalized orthomodular lattices . . . . . . . . . . ........

L. Carlitz, A three-term relation for some sums related to Dedekind sums . . . . . . . . . .....

Arthur Herbert Copeland, Jr. and Albert Oscar Shar, Images and pre-images of localization

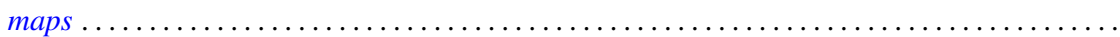

G. G. Dandapat, John L. Hunsucker and Carl Pomerance, Some new results on odd perfect

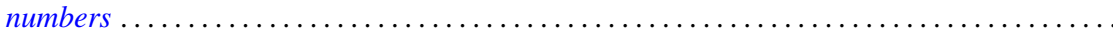

M. Edelstein and L. Keener, Characterizations of infinite-dimensional and nonreflexive

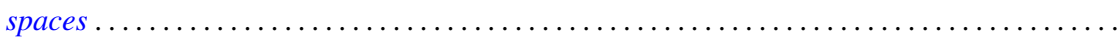

Francis James Flanigan, On Levi factors of derivation algebras and the radical embedding

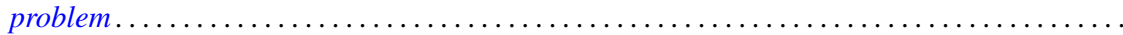

Harvey Friedman, Provable equality in primitive recursive arithmetic with and without

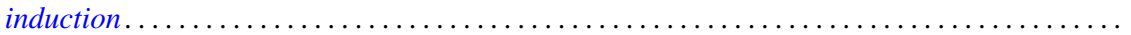

Joseph Braucher Fugate and Lee K. Mohler, The fixed point property for tree-like continua with

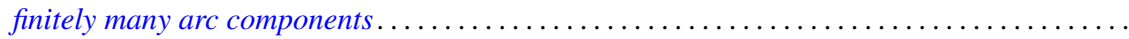

John Norman Ginsburg and Victor Harold Saks, Some applications of ultrafilters in

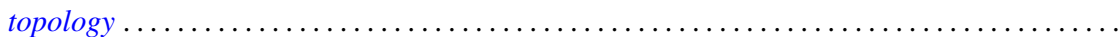

Arjun K. Gupta, Generalisation of a "square" functional equation .....................

Thomas Lee Hayden and Frank Jones Massey, Nonlinear holomorphic semigroups ..........

V. Kannan and Thekkedath Thrivikraman, Lattices of Hausdorff compactifications of a locally

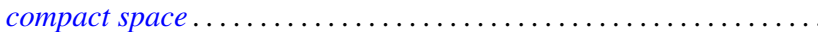

J. E. Kerlin and Wilfred Dennis Pepe, Norm decreasing homomorphisms between group

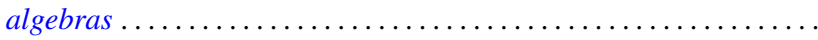

Young K. Kwon, Behavior of $\Phi$-bounded harmonic functions at the Wiener boundary ...

Richard Arthur Levaro, Projective quasi-coherent sheaves of modules .

Chung Lin, Rearranging Fourier transforms on groups...........................

David Lowell Lovelady, An asymptotic analysis of an odd order linear differential equation . . 4475

Jerry Malzan, On groups with a single involution .......................... 481

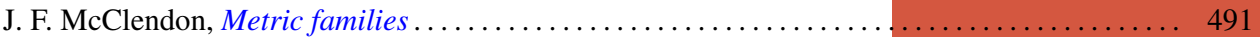

Carl Pomerance, On multiply perfect numbers with a special property .

Mohan S. Putcha and Adil Mohamed Yaqub, Polynomial constraints for finiteness of semisimple rings. .

Calvin R. Putnam, Hyponormal contractions and strong power convergence . . . . . . . . . 531

Douglas Conner Ravenel, Multiplicative operations in $\mathrm{BP} * \mathrm{BP} \ldots \ldots \ldots \ldots \ldots \ldots \ldots \ldots \ldots .539$

Judith Roitman, Attaining the spread at cardinals which are not strong limits . . . . . . . . . 545

Kazuyuki Saitô, Groups of *-automorphisms and invariant maps of von Neumann algebras . . . 553

Brian Kirkwood Schmidt, Homotopy invariance of contravariant functors acting on smooth

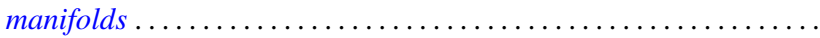

Kenneth Barry Stolarsky, The sum of the distances to $N$ points on a sphere.

Mark Lawrence Teply, Semiprime rings with the singular splitting property.

J. Pelham Thomas, Maximal connected Hausdorff spaces..............

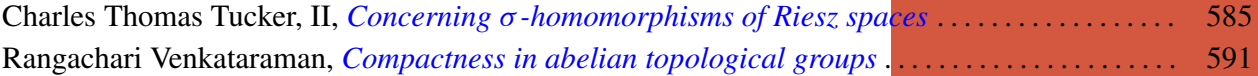

William Charles Waterhouse, Basically bounded functors and flat sheaves . . . . . . . . . . . 597

David Westreich, Bifurcation of operator equations with unbounded linearized part ......... 611

William Robin Zame, Extendibility, boundedness and sequential convergence in spaces of 